\title{
VACUUM ENERGY: IF NOT NOW, THEN WHEN?
}

Sidney A. Bludman ${ }^{\mathrm{a}}$ *

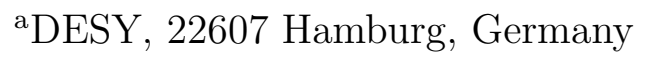

University of Pennsylvania, Philadelphia, PA 19104

We review the cosmological evidence for a low matter density universe and a cosmological constant or dynamical vacuum energy and address the cosmological coincidence problem: why is the matter density about one-half the vacuum energy now. This is reasonble, following the anthropic argument of Efstathiou and of Martel, Schapiro \& Weinberg.

\section{DENSITY OF RADIATION AND CLUSTERED MATTER IS LOW $[1,2]$}

\subsection{Different Kinds of Dark Matter and Vacuum Energy}

Radiation, massive neutrinos, luminous matter are now known to consitute the least part of the Universe: $\Omega_{\text {rad }}=5 \times 10^{-5} h^{-2}, \Omega_{\nu}=(0.003-0.15) \sim \Omega_{\star}=(0.005 \pm 0.002)$. Instead, we find most of the Universe in three forms:

- Baryonic Matter: $\Omega_{B}=(0.019 \pm 0.0012) h^{-2} \sim(0.05 \pm 0.005)>>\Omega_{\star}$

- Non-baryonic Cold Dark Matter: $\Omega_{m}=(0.35 \pm 0.1) h^{-1 / 2} \sim(0.4 \pm 0.1)>>\Omega_{B}$

- Smooth Energy: $\Omega_{X} \sim 0.6 \pm 0.2$.

Here the various $\Omega_{i}$ are the fractional contributions to the present critical density $\rho_{c r} \equiv$ $3 H_{0}^{2} / 8 \pi G$.

From the cosmic background radiation (CBR), we know that the spatial curvature $\Omega_{k} \equiv 1-\Omega_{m}-\Omega_{X} \pm 0.2$, a flat universe of critical density, dominated by non-baryonic matter and smooth energy. Inflation explains this flatness of the present universe, as a consequence of an early de Sitter phase making an old universe, now expanding slowly.

We distinguish two kinds of dark matter relicts of the Big Bang: (1) Hot Dark Matter (HDM), light neutrinos $\sum m_{\nu}<45 \mathrm{eV}$. Because we know large-scale structure (LSS) evolved hierarchically, $\Omega_{\nu}<0.15$, HDM cannot dominate cosmologically; (2)Instead, Cold Dark Matter (CDM), say $10^{-5 \pm 1} \mathrm{eV}$ axions or $(50-500) \mathrm{GeV}$ neutralinos must seed the gravitational clustering that we see. Indeed, $2 / 3$ of our Galactic halo DM cannot be baryonic, and must be CDM.

\subsection{The Density of Clustered Matter: $\Omega_{m} \sim 0.4$}

The observed primordial abundances of the four light nulei are concordant with a single baryon density

$$
\Omega_{B}=(0.019 \pm 0.0012) h^{-2}=0.05 \pm 0.01
$$


This value is consistent with $\Omega_{B} h^{2}>0.015$ from large scale structure (LSS) and with the height of the first acoustic peak in cosmic background radiation angular distribution.

In rich clusters of galaxies, most baryons reside in the hot inter-galactic gas and and reveal themselves in inner bremsstrahlung X-rays and in Sunyaev- Zeldovich infra-red radiation. These two sources determine the total baryon fraction to be $f_{B}=(0.075 \pm$ $0.002) h^{-3 / 2}$ and $(0.06 \pm 0.006) h^{-1}$ respectively. Together with the above value of $\Omega_{B}$, this determines the clustered mass density [3]

$$
\Omega_{m} \equiv \Omega_{B} / f_{B}=(0.4 \pm 0.1)
$$

The simplest argument for mean background mass density $\Omega_{m}>0.3(95 \%$ C.L.) derives from limits on the outflow from cosmic voids. Supporting evidence also come from

- The evolution of the number density of rich clusters $\Omega_{m}=(0.45 \pm 0.1)$;

- Peculiar velocities of IRAS galaxies;

- Large Scale Structure, which imprints the present mass density power spectrum, showing $\Omega_{m} h=(0.25 \pm 0.05)$;

- $L y-\alpha$ absorption spectra compared with LSS simulations when growth was becoming non-linear, showing $\Omega_{m}=0.34 \pm 0.1$.

\section{ACCELERATED EXPANSION REQUIRES VACUUM ENERGY}

Different kinds of energy density with equation of state $w \equiv P / \rho$, expand adiabatically with scale $a$ at different rates $\rho \sim a^{-n}, n \equiv 3(1+w)$ :

\begin{tabular}{|l|c|c|}
\hline substance & $w$ & $n$ \\
\hline radiation & $1 / 3$ & 4 \\
NR matter & 0 & 3 \\
curvature & $-1 / 3$ & 2 \\
vacuum & -1 & 0 \\
\hline
\end{tabular}

In this list, we have also included the spatial curvature term which falls off as $a^{-2}$, just as a substance with $\rho+3 P=0$.

\subsection{Expansion Age Requires Vacuum Energy}

The oldest globular clusters are aged 11.5 \pm 1.3 Gyr. Allowing about 1 Gyr before star formation at $z \sim 5$, this gives a dynamical age $t_{0} \geq 14$ Gyr. Because the Hubble constant is now known $H_{0}=73 \pm 6$ (stat) \pm 8 (syst), $H_{0} t_{0} \geq 0.93 \pm 0.16$. The $1 \sigma$ limits rule out an Einstein-deSitter $\Omega_{m}=1, \Omega_{\Lambda}=0$ flat universe, but are consistent with a low matter density $\Omega_{m} \sim 0.4$ and a cosmological constant $0.3<\Omega_{\Lambda}<1.3$. The two extreme $\Omega_{m}=0.4$ geometries, open $\left(\Omega_{\Lambda}=0, H_{0} t_{0}=0.80\right)$ and flat $\left(\Omega_{\Lambda}=0.6, H_{0} t_{0}=0.89\right)$, are distinguishable by different cosmographic distance measurements:

- A difference in lumininosity distance, observable with SN Ia;

- Different comoving volume elements, observable in the number density of gravitational lenses;

- Different differential intersection probabilities, observable in QSO absorption lines or pencil-beam redshift surveys. 
We now want to fix the present values of the two cosmological parameters, $\Omega_{\Lambda}$ and $\Omega_{m}$ separately.

\subsection{The Young Universe Was Expanding Slower Than Now}

A selected sample of supernovae Ia ("Branch normal") are "standard bombs", from which the luminosity distance $d_{L}$ can be measured at high red-shift $z>0.4$. From more than 40 such SN Ia, the combination $\Omega_{\Lambda}-4 \Omega_{m} / 3=1 / 3 \pm 1 / 6$ is measured $[4,5]$. The nearly orthogonal combination $\Omega_{m}+\Omega_{\Lambda} \sim 1$, gives the joint fit $\Omega_{m} \sim 1 / 3, \quad \Omega_{\Lambda} \sim 2 / 3$. More precisely, what is established is that the present deceleration $q_{0}=\left(\Omega_{m}+(1+\right.$ $\left.\left.3 w_{X}\right) \Omega_{X}\right) / 2<0$, because of a component $X$, with $w_{X}<-1 / 2$. This shows that a positive pressure $w>0$ would not cause, but retard, cosmic expansion. Any exotic component with $w_{X}<-1 / 3$ violates the once-respected energy positivity condition $\rho+3 P<0$, and accelerates expansion, acting as a negative (accelerating) active gravitational mass. Weaker supporting evidence comes from double-lobe radio galaxies, from rich clusters, and from the frequency of multiply-lensed QSOs.

Because the supernovae observations are so important, we must remember that what is observed is only that high redshift supernova are fainter by 0.25 magnitude than they would appear in an accelerating universe. Possible systematic errors that might produce the same apparent (13\%) dimming are

- Part of the dimming may be caused by intervening (gray!) dust;

- Nearby supernovae may have brightened with age. We now need more nearby standard Ia 's for calibration.

\section{QUINTESSENCE: DYNAMIC VACUUM ENERGY}

The present supernovae data does not require a cosmological constant $\rho_{\Lambda}=-P_{\Lambda}=$ constant, but only some unclustered smooth dark energy or quintessence, with $w_{X}<$ $-1 / 2$ [2]. This "time-dependent vacuum energy", may be no more than a scalar field

$\rho=(1 / 2) \dot{\phi}^{2}+V(\phi), P=(1 / 2) \dot{\phi}^{2}-V(\phi)$ slowly rolling down towards its true ground state. If the potential energy $V(\phi) \geq$ thrice the kinetic energy, the time averaged $-1<$ $w_{\text {eff }}<-1 / 2$, and $\rho_{X}$ diminishes slower than $a^{-3 / 2}$ and, since $z<(0.14-0.3)$, already dominates the curvature and matter energy density.

Such a scalar field is reminiscent of the McCrae-Hoyle C-field, with two important differences: (1) Steady state cosmology requires continuous creation, violates local energy conservation and is ruledd out by baryogenesis, nucleogenesis, cosmic background radiation and the observed evolution of galaxies and QSOs; (2) Quintessence is consistent with the conservation laws of General Relativity.

\section{CONCLUSIONS: $\Omega_{X} \sim \Omega_{m}$ IS REASONABLE FOR OUR UNIVERSE}

Inflation never determined the matter and vacuum energy content of the universe, other than requiring $\rho_{m}+\rho_{\Lambda}=\rho_{c r}$. There (fortunately) never was any theory predicting $\Lambda=0$, nor any symmetry principle protecting this value: a rolling scalar field or any value $\Omega_{\Lambda}<1$ is reasonable. The real cosmological problem is not why $\rho_{\Lambda}$ or $\rho_{X}<1$ are present, but to explain the cosmic cooincidence, that the matter density $\rho_{m}$, which is ever-diminishing has the value $\sim \Omega_{\Lambda} / 2$ now. 
While we hope for a future theory of everything that will calculate all the constants of particle physics, $\Omega_{\Lambda}$, alone of all constants, may not be calculable from first principles. Instead, we ask what values for $\Omega_{\Lambda} / \Omega_{m}$ would be reasonable cosmologically.

\section{1. "If not now, then when?" [6]}

Efstathiou [7] and Martel, Shapiro \& Weinberg [8] answered this question, before the supernovae observations establishing a constant or time-varying vacuum energy. If $\Omega_{\Lambda}$ is a stochastic variable, the probability distribution of its observed values should be proportional to the fraction of matter destined to condense into galaxies, stars, astronomers. They find that the probability distribution for random living astronomers observing a value $\Omega_{\Lambda}$, in a nearly flat universe, is broadly distributed about a median $\Omega_{\Lambda} \sim 0.75$ : (1) If $\Omega_{\Lambda} / \Omega_{m}>4$, the universe would have expanded too fast to form such structures; (2) $\Omega_{\Lambda} / \Omega_{m}<0.02$ is possible, but relatively unlikely; (3) Values $\Omega_{\Lambda} / \Omega_{m} \sim 2$ would be observed $5-12 \%$ of the time. The observed value, while below the median in probability, is close to the maximum value consistentwith the formation of galaxies by the present epoch.

\subsection{The Future}

We live at the only time we could be living, at the end of the epoch of galaxy and star formation. Barring another (unlikely) particle physics phase transition, the universe is moving monotonically toward a de Sitter universe fixed point.

\subsection{The Nature of Scalar Fields}

Higgs scalars and vacuum expectation values and masses enter mysteriously into presentday broken symmetry gauge theories. A rolling scalar field with very low mass $\left(<10^{-3} \mathrm{eV}\right)$ is an important mediator of the longest range forces, which may be telling us something: the anthropic principle recognized in cosmology, may become the heuristic guide particle physics needs.

\section{REFERENCES}

1. M.S. Turner, Proc. Particle Physics and the Universe (Cosmo-98), (ed. D.O. Caldwell, AIP)

2. L. Wang, R.R. Caldwell, J.P. Ostriker and P.J. Steinhardt, Ap. J. (submitted), astroph/9901388, (1999).

3. S.A. Bludman, Ap. J. 508, 535 (1998).

4. S. Perlmutter, et al, LBL-42230 (1998), astro-ph/9812473; S. Perllmutter et al, Ap. J (in press), astro-ph/981233.

5. A.G. Riess et al, Astron. J. 116, 1009 (1998); P.M. Garnavich et al, Ap. J. 509, 74 (1998).

6. Rabbi Hillel, Sayings of the Fathers, 1:14, c.60 B.C.-A.D. 9?.

7. G. Efstathiou, M.N.R.A.S. 274, 229 (1995).

8. H. Martel, P.R. Shapiro and S. Weinberg, Ap. J. 492, 29 (1998). 